\title{
Characterizing the dynamical phase diagram of the Dicke model via classical and quantum probes
}

\author{
R. J. Lewis-Swan $\odot,,^{1,2}$ S. R. Muleady $\odot,{ }^{3,4, *}$ D. Barberena $\odot, 3,4, *$ J. J. Bollinger $\odot, 5$ and A. M. Rey $\oplus^{3,4}$ \\ ${ }^{1}$ Homer L. Dodge Department of Physics and Astronomy, The University of Oklahoma, Norman, Oklahoma 73019, USA \\ ${ }^{2}$ Center for Quantum Research and Technology, The University of Oklahoma, Norman, Oklahoma 73019, USA \\ ${ }^{3}$ JILA, NIST, Department of Physics, University of Colorado, Boulder, Colorado 80309, USA \\ ${ }^{4}$ Center for Theory of Quantum Matter, University of Colorado, Boulder, Colorado 80309, USA \\ ${ }^{5}$ National Institute of Standards and Technology, Boulder, Colorado 80305, USA
}

(Received 8 February 2021; accepted 11 May 2021; published 4 June 2021)

\begin{abstract}
We theoretically study the dynamical phase diagram of the Dicke model in both classical and quantum limits using large, experimentally relevant system sizes. Our analysis elucidates that the model features dynamical critical points that are strongly influenced by features of chaos and emergent integrability in the model. Moreover, our numerical calculations demonstrate that mean-field features of the dynamics remain valid in the exact quantum dynamics, but we also find that in regimes where quantum effects dominate signatures of the dynamical phases and chaos can persist in purely quantum metrics such as entanglement and correlations. Our predictions can be verified in current quantum simulators of the Dicke model including arrays of trapped ions.
\end{abstract}

DOI: 10.1103/PhysRevResearch.3.L022020

Introduction. Advances in atomic, molecular, and optical (AMO) quantum simulators are driving a surge in the investigation of dynamical phase transitions (DPTs) and associated nonequilibrium phases of matter [1-6]. In a closed system, a DPT is a critical point separating distinct dynamical behaviors that emerge after a sudden quench of a control parameter [7-13], and can be defined via nonanalytic behavior in a time-averaged order parameter [14-17].

To date, the majority of experimental investigations in this direction have been tailored towards integrable models, featuring effective infinite-range interactions between spins, which admit analytical treatments [18-21]. Richer nonintegrable models have been pursued in trapped ion systems [22], but the associated complexity of the quantum dynamics limited the theoretical analysis of the DPT to small system sizes and prevented a rigorous scaling analysis. Hence, it is highly desirable to find and study DPTs in nonintegrable models featuring novel nonequilibrium phenomena that are both implementable in tunable quantum simulators and theoretically tractable under controllable approximations.

We advance this direction by studying a DPT in the iconic Dicke model [23-25], which describes the collective coupling of many spins to a single harmonic oscillator. The model is attractive as it features an array of phenomena, such as nonintegrability [26-28], chaos [29-31], and equilibrium quantum phase transitions (QPTs) in both ground and excited states [32-37], but involves only a pair of disparate degrees of

\footnotetext{
*These two authors contributed equally.

Published by the American Physical Society under the terms of the Creative Commons Attribution 4.0 International license. Further distribution of this work must maintain attribution to the author(s) and the published article's title, journal citation, and DOI.
}

freedom such that the model remains amenable to analytic and numerical treatments. Moreover, the model is already studied in state-of-the-art AMO quantum simulators, including trapped-ion arrays [38,39] and cavity QED [40-43]. Here, we investigate the DPT in analytically tractable spin- and bosondominated limits, as well as a nonintegrable regime where near-resonant coupling of spin and bosons leads to chaotic dynamical phases also seen in other non-integrable systems $[7,8]$. Following recent works linking DPTs to coexisting excited-state QPTs (EQPTs) [20,36,44], we find different dynamical critical points in the spin and boson-dominated regimes that reflect the presence of distinct EQPTs in these limits. By studying large, experimentally relevant system sizes using efficient numerical methods we are able to demonstrate that mean-field features of the dynamics remain valid in the exact quantum dynamics. Conversely, in regimes where quantum effects dominate we find that signatures of the DPT and chaos persist in purely quantum metrics such as entanglement and correlations.

Model. The Dicke Hamiltonian for $N$ spin-1/2 particles is given by [30]

$$
\hat{H}_{\mathrm{D}}=\frac{2 g}{\sqrt{N}}\left(\hat{a}+\hat{a}^{\dagger}\right) \hat{S}_{z}+\delta \hat{a}^{\dagger} \hat{a}+\Omega \hat{S}_{x} .
$$

Here, $\hat{a}\left(\hat{a}^{\dagger}\right)$ is the bosonic annihilation (creation) operator of an oscillator with frequency $\delta, \hat{S}_{\alpha}=1 / 2 \sum_{j=1}^{N} \hat{\sigma}_{j}^{\alpha}$ are collective spin operators for $\alpha=x, y, z$, and $\hat{\sigma}_{j}^{\alpha}$ Pauli matrices of the $j$ th spin. The spins are subject to a transverse field of strength $\Omega$ and the spin-boson coupling is characterized by g. The Hamiltonian exhibits a $\mathbb{Z}_{2}$ parity symmetry associated with the operator $\hat{\Pi}=e^{i \pi\left(\hat{S}_{x}+\hat{a}^{\dagger} \hat{a}+N / 2\right)}$ such that $\left[\hat{H}_{\mathrm{D}}, \hat{\Pi}\right]=0$.

The equilibrium phase diagram of the Dicke model features a ground-state QPT at a critical coupling $g_{\mathrm{QPT}}=$ $\sqrt{\delta \Omega} / 2[32,33]$, which delineates a superadiant $\left(g \gg g_{\mathrm{QPT}}\right)$ 


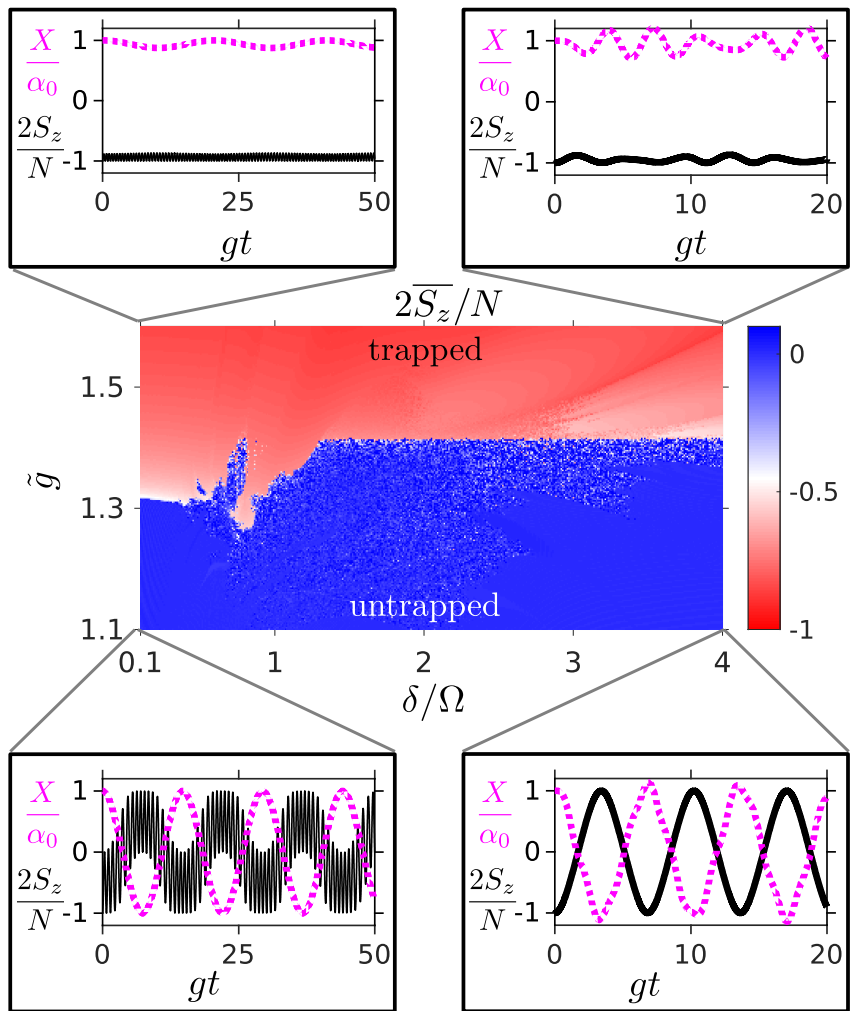

FIG. 1. Dynamical phase diagram for time-averaged order parameter $\overline{S_{z}}$ (center) and typical time traces (surrounding) for the Dicke model. Parameters of time traces are (clockwise from top left) $(\delta / \Omega, \tilde{g})=(0.1,2),(\delta / \Omega, \tilde{g})=(4,2),(\delta / \Omega, \tilde{g})=(4,1)$, and $(\delta / \Omega, \tilde{g})=(0.1,1)$. Time-averaged $\overline{S_{z}}$ is obtained up to a maximum time $g T=10^{4}$.

phase, with degenerate ground states associated with different parity sectors that in the limit $\Omega \rightarrow 0$ take the form $\left|\psi_{s}\right\rangle=$ $\frac{1}{\sqrt{2}}\left[\left|(-N / 2)_{z}\right\rangle \otimes\left|\alpha_{s}\right\rangle \pm\left|(-N / 2)_{z}\right\rangle \otimes\left|-\alpha_{s}\right\rangle\right]$, and a normal $\left(g \ll g_{\text {QPT }}\right)$ phase, with $\left|\psi_{n}\right\rangle \approx\left|(N / 2)_{x}\right\rangle \otimes|0\rangle$ in the limit of large $\Omega$ [38]. Here, we have defined collective spin states via $\hat{S}_{x, y, z}\left|m_{x, y, z}\right\rangle=m_{x, y, z}\left|m_{x, y, z}\right\rangle$ and $\left| \pm \alpha_{s}\right\rangle$ is the bosonic coherent state for $\alpha_{s}=g \sqrt{N} / \delta$. Additionally, an excited-state QPT (EQPT) exists in the spectrum of the superradiant phase, $g>$ $g_{\mathrm{QPT}}$, defined by a critical energy $E_{c}=-\Omega N / 2[34,36,37]$. The EQPT is signaled by a nonanalyticity in the density of states [35] and the existence of pairs of degenerate eigenstates with different parity for $E<E_{c}$.

Dynamical phase diagram. We study the dynamical phase diagram that arises after a quench of the transverse field. Concretely, the system is initialized in the ground state of $\hat{H}_{\mathrm{D}}$ at fixed $g$ and $\delta$ with $\Omega=0$, such that $|\psi(0)\rangle=\left|(-N / 2)_{z}\right\rangle \otimes$ $\left|\alpha_{s}\right\rangle$, and the transverse field is then quenched to a final value $\Omega \neq 0$. To garner insight into the dynamics we first study the classical model (Figs. 1-3) before probing the role of quantum fluctuations. The classical limit of the Dicke model is equivalent to solving the Heisenberg equations of motion for operators under a mean-field approximation, wherein expectation values are factorized according to $\left\langle\hat{\mathcal{O}}_{1}(t) \hat{\mathcal{O}}_{2}(t)\right\rangle=$ $\left\langle\hat{\mathcal{O}}_{1}(t)\right\rangle\left\langle\hat{\mathcal{O}}_{2}(t)\right\rangle$ [45]. For brevity we adopt the notation $\mathcal{O} \equiv$ $\langle\hat{\mathcal{O}}(t)\rangle$ herein.
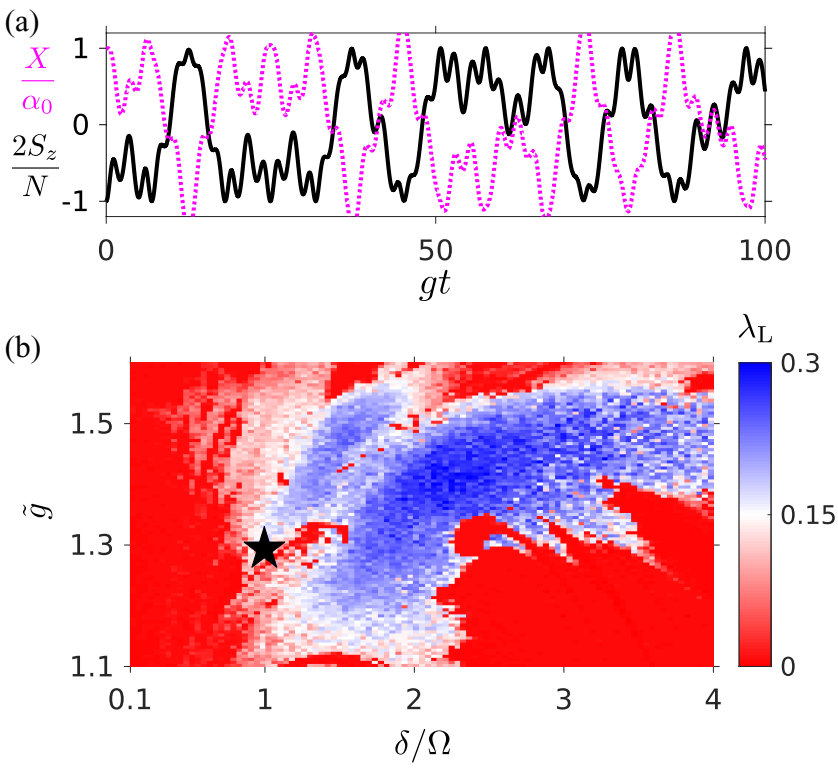

FIG. 2. (a) Typical time trace near the transition in the resonant region, $\tilde{g} \approx 1.299$ and $\delta / \Omega=1$. (b) Characterization of phase space via Lyapunov exponent $\lambda_{\mathrm{L}}$. The star indicates parameter regime of the time trace.

(a)
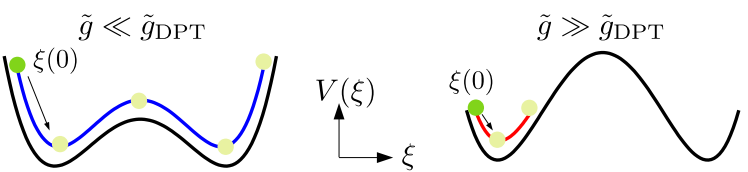

(b) $i$

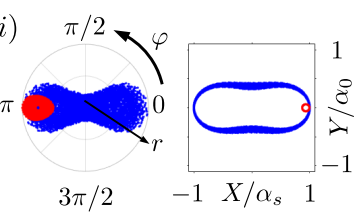

(c)
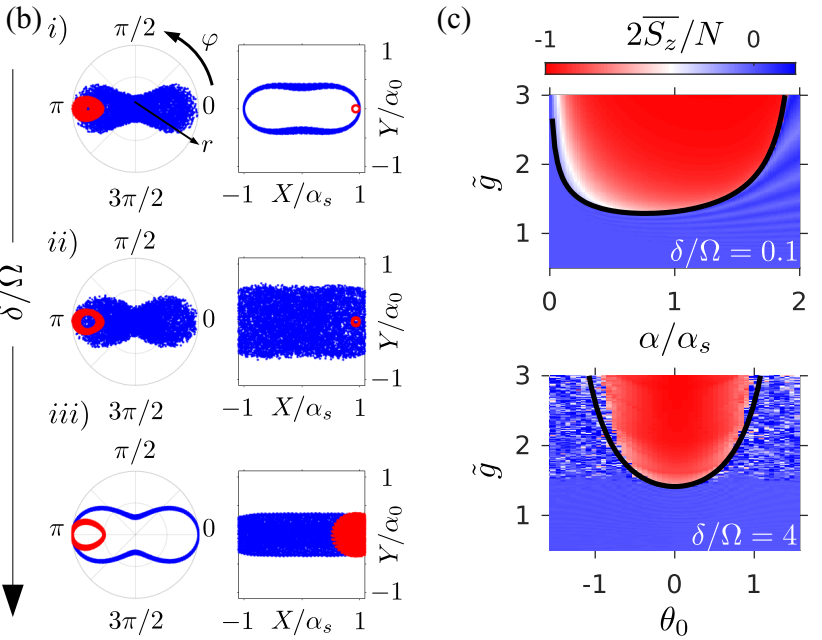

FIG. 3. (a) In the integrable limits the DPT can be described as a particle (green markers) in a $1 \mathrm{D}$ potential with coordinate $\xi=S_{z}$ $(\delta / \Omega \gg 1)$ or $\xi=X(\delta / \Omega \ll 1)$. At small $\tilde{g} \ll \tilde{g}_{\text {DPT }}$ (untrapped phase, left) the particle has sufficient energy to traverse both wells of the potential, whereas for large $\tilde{g} \gg \tilde{g}_{D P T}$ (trapped phase, right) the particle remains energetically confined to a single well. (b) Typical trajectories in phase space for trapped (red) and untrapped (blue) phases at $\delta / \Omega=(0.1,1,10)$ (top to bottom). For the spin phasespace we use coordinates $r=1+2 S_{x} / N$ and $\varphi=\arctan \left(S_{y} / S_{z}\right)$ (c) Dynamical phase diagram as a function of initial state. At small detuning $\delta / \Omega=0.1$ we vary the boson amplitude $\alpha$ (top panel), and at large detuning $\delta / \Omega=4$ we vary the tipping angle $\theta_{0}$ relative to the south pole of the Bloch sphere $\left(S_{z}=-N / 2\right)$ (bottom panel). 
It is convenient to study dynamics in terms of two variables: $\tilde{g}=2 g / \sqrt{\delta \Omega} \equiv g / g_{\mathrm{QPT}}$ and $\delta / \Omega$. The former characterizes the effective strength of the spin-boson interaction relative to the single-particle terms, while the latter describes the relative energy scales of spin and bosonic excitations and loosely expresses the relative importance of each degree of freedom to the dynamics. In the limit $\delta / \Omega \gg 1$ and $\tilde{g} \sim 1$ the dynamics are equivalent to a spin model described by the Lipkin-Meshkov-Glick (LMG) Hamiltonian, $\hat{H}_{\text {eff }}=(\chi / N) \hat{S}_{z}^{2}+\Omega \hat{S}_{x}$ with the boson-mediated interaction characterized by $\chi \equiv 4 g^{2} / \delta[17,46]$. For $\delta / \Omega \ll 1$ and $\tilde{g} \sim 1$ the spins are instead slaved to the dynamics of the dominant bosons [45]. However, in this limit a simple boson-only Hamiltonian is not generally applicable. Lastly, for $\delta / \Omega \sim 1$ the dynamics is complicated and involves both degrees of freedom. Herein, we refer to the integrable limits $\delta / \Omega \gg$ 1 and $\delta / \Omega \ll 1$ as the spin-dominated (SDR) and bosondominated (BDR) regimes respectively, and the nonintegrable case of $\delta / \Omega \sim 1$ as the resonant regime (RR).

The typical dependence of the dynamics on $\tilde{g}$ is shown in the time traces of Fig. 1 for SDR and BDR. In both limits we identify that the dynamics can be characterized as either trapped or untrapped. The former occurs when the spin-boson interaction dominates the Hamiltonian and leads to a locking of the spins and bosons close to their initial configuration. In the SDR this has been interpreted as a self-generated detuning $\propto\left\langle\hat{S}_{z}\right\rangle \hat{S}_{z}$ that locks out rotations due to the transverse field [21]. Conversely, the untrapped dynamics are characterized by large coherent oscillations in $S_{z}$ and $X=\frac{1}{2}\left\langle\hat{a}+\hat{a}^{\dagger}\right\rangle$ dominated by the single-particle terms of the Hamiltonian.

We classify the dynamical phases and identify a DPT using a pair of interchangeable time-averaged order parameters $\overline{S_{z}}=$ $\lim _{T \rightarrow \infty}(1 / T) \int_{0}^{T} S_{z}(t) d t$ and $\bar{X}=\lim _{T \rightarrow \infty}(1 / T) \int_{0}^{T} X(t) d t$. The trapped phase is defined by nonzero $\overline{S_{z}} \neq 0$ and $\bar{X} \neq$ 0 , while in the untrapped phase $\overline{S_{z}}=\bar{X}=0$. The phases are separated by a critical coupling (main panel of Fig. 1): (i) $\tilde{g}_{\mathrm{DPT}}^{s} \approx \sqrt{2}$ in the spin-dominated regime and (ii) $\tilde{g}_{\mathrm{DPT}}^{b} \approx$ $3^{1 / 4}$ in the boson-dominated regime. The dynamical phases in the LMG model have recently been observed in a cavity-QED quantum simulator [21].

In the nonintegrable $\mathrm{RR}, \delta / \Omega \sim 1$, the dynamical phase diagram is more complex. The main panel of Fig. 1 illustrates that the time-averaged order parameter becomes noisy in the untrapped phase. In this regime typical time traces [Fig. 2(a)] feature erratic oscillations in both spin and boson observables and there exist short periods where the system abruptly becomes re-trapped. This behavior signals a chaotic dynamical phase $[7,8]$ that arises due to the known nonintegrability of the Dicke model for $\tilde{g}>1$ and $\delta \sim \Omega[27,29,30,32]$.

We support this by computing the Lyapunov exponent $\lambda_{\mathrm{L}}$ [30] in Fig. 2 (see also Ref. [45]), as a function of $\tilde{g}$ and $\delta / \Omega$ for the initial condition corresponding to $|\psi(0)\rangle$. Chaos, and thus nonintegrability, is signalled by $\lambda_{\mathrm{L}}>0$ [47] in regions of parameter space that qualitatively coincide with the noisy behavior of $\overline{S_{z}}$ (Fig. 1). We also find evidence of small regions where the chaotic dynamical phases exist but $\lambda_{L} \rightarrow 0$ (within numerical error). This is not contradictory as the underpinning requirement for the so-called chaotic dynamical phase to exist is in fact the non-integrability of the Dicke model due to the coupling of the spin and boson degrees of freedom.

The dynamical phase diagram of SDR and BDR is captured by an effective model involving only the dominant degree of freedom [45,48-52]. Specifically, the mean-field dynamics are reduced to an equivalent picture of a classical particle with coordinate $\xi=S_{z}$ (spin dominated) or $\xi=X$ (boson dominated) confined within a one-dimensional (1D) potential $V(\xi)$ that depends only on $\tilde{g}, \delta / \Omega$, and the initial state. Near the DPT $V(\xi)$ is well approximated by a double well potential [Fig. 3(a)].

The initial position and velocity of the particle, $\xi(0)$ and $\dot{\xi}(0)$, and the relative height of the central maximum, $V(0)$, characterize the dynamics of the system [8]. For $\tilde{g}>\tilde{g}_{\text {DPT }}$ and the particle initially located in one well, $\xi(0) \neq 0$, the particle has insufficient mechanical energy to overcome the barrier and remains confined. On the other hand, for $\tilde{g}<\tilde{g}_{\text {DPT }}$ the particle has sufficient energy to pass over the barrier and traverses freely between both wells. The former scenario describes trapped dynamics, $\bar{\xi} \neq 0$, while the latter describes the untrapped phase, $\bar{\xi}=0$. The critical point is defined as the condition for which the particle first surpasses the central barrier and we find $\tilde{g}_{\mathrm{DPT}}^{s}=\sqrt{2}$ and $\tilde{g}_{\mathrm{DPT}}^{b}=3^{1 / 4}$, respectively, in agreement with Fig. 1.

The DPT in the SDR coincides with the well known EQPT of the Dicke Hamiltonian [34,35,37], i.e., the energy of the initial state matches the EQPT critical energy, $E_{0} \equiv$ $\left.\left\langle\psi_{0}|\hat{H}| \psi_{0}\right\rangle\right|_{\tilde{g}_{\mathrm{DPT}}^{s}}=E_{c}$. The EQPT features a nonanalyticity in eigenstate observables at $E_{c}$ that can be intimately related to the behavior of $\bar{S}_{z}$ at the DPT and is related to a saddle point that emerges in the classical phase space [35,37]. In the BDR the Dicke model has been predicted to feature a set of EQPTs at energies $\left\{E_{c}^{(b)}\right\}$ that arise in distinct sectors of the energy spectrum labeled by values of an emergent conserved quantity, which restores the integrability of the model [51,52]. In the large $N$ limit our state occupies a single sector of the spectrum and at $\tilde{g}_{\text {DPT }}^{b}<\tilde{g}_{\text {DPT }}^{s}$ the energy of the initial state $\left.E_{0} \equiv\left\langle\psi_{0}|\hat{H}| \psi_{0}\right\rangle\right|_{\tilde{g}_{\mathrm{DPT}}^{b}}$ matches a different EQPT critical energy $E_{c}^{(b)}=-\sqrt{3} \Omega N / 4$, which is consistent with the observed shift in the DPT critical point.

The potential model is further evidenced by inspecting typical trajectories of trapped and untrapped cases in the classical phase space, as shown in Fig. 3(b) for the three regimes. In BDR and SDR we observe well defined orbits in the dominant degree of freedom that are centered around fixed point(s) in phase space corresponding to the minima of the potential well(s) [45]. The trajectories of the complementary slaved observables show similar behavior, but tend to densely fill out the available phase space due to fast micromotion on top of the slower orbits arising from the enslavement to the dominant degree of freedom (see also Fig. 1). In the resonant regime the potential description breaks down as no single degree of freedom dominates. Consequently, we observe a lack of clear orbits in phase space, although the erratic trajectories do fill out distinct volumes of phase space in each dynamical phase.

DPTs in isolated systems are intrinsically dependent on initial conditions [21]. We demonstrate this by probing initial states of the form $|\psi(0)\rangle=\left|(-N / 2)_{\mathbf{n}}\right\rangle \otimes|\alpha\rangle$, where $\alpha \in$ 

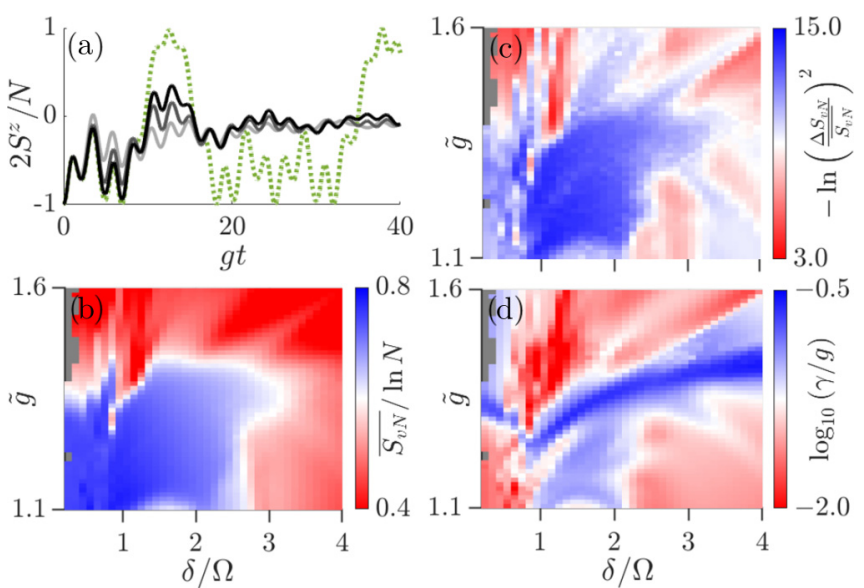

FIG. 4. Signatures of DPT in quantum dynamics (see Ref. [45] for details). (a) Time traces of quantum dynamics in the resonant regime, $\tilde{g}=1.3$ and $\delta / \Omega=1$, for $N=40$ (light gray), 200 (gray), and 600 (black). The mean-field solution (green) is shown for reference. (b) Time-averaged entanglement entropy $\overline{S_{\mathrm{vN}}}=(1 / T) \int_{t_{0}}^{T+t_{0}} d t S_{\mathrm{vN}}(t)$ for $S_{\mathrm{vN}}=-\operatorname{Tr}\left[\hat{\rho}_{s} \ln \left(\hat{\rho}_{s}\right)\right]$ where $\hat{\rho}_{s}$ is the reduced density matrix of the spins. (c) Temporal fluctuations of entanglement entropy, $\left(\Delta S_{\mathrm{vN}}\right)^{2}=(1 / T) \int_{t_{0}}^{T+t_{0}} d t\left[S_{\mathrm{vN}}(t)-\overline{S_{\mathrm{vN}}}\right]^{2}$, normalized by $\overline{S_{\mathrm{vN}}}$. (d) Growth rate $\gamma$ of quantum fluctuations, obtained by an empirical fit to $\left\langle\left(\Delta \hat{S}_{z}\right)^{2}\right\rangle \propto\left(1-e^{-\gamma t}\right)$. Data in (b)-(d) are obtained for $N=600$. Time averages in (b) and (c) are computed over a window $t \in\left[t_{0}, T+t_{0}\right]$ for $g t_{0}, g T=150$, so as to reduce transient effects in steady state estimates. Gray regions correspond to excluded data.

$\mathbb{R}$ and we define $\hat{S}_{\mathbf{n}}\left|m_{\mathbf{n}}\right\rangle=m_{\mathbf{n}}\left|m_{\mathbf{n}}\right\rangle$ for $\hat{S}_{\mathbf{n}}=\hat{\mathbf{S}} \cdot \mathbf{n}$ with $\mathbf{n}=$ $\left(0, \sin \left(\theta_{0}\right), \cos \left(\theta_{0}\right)\right)$ defined by the tipping angle $\theta_{0}$ from the south pole of the collective Bloch sphere. In Fig. 3(c) we probe the dependence of $\tilde{g}_{\mathrm{DPT}}$ on the initial amplitude $\alpha$ in the BDR, while in the SDR we vary the initial tipping angle $\theta_{0}$. We observe good agreement with analytic predictions for $\tilde{g}_{\text {DPT }}$ based on the potential model [45]. Small deviations are observed for $\theta \approx \pm \pi / 2$ and $\alpha / \alpha_{s} \rightarrow 0$, which correspond to initializing a particle near the central maximum of the potential such that even a small off-resonant exchange of energy with the complementary degree of freedom is enough for the particle to overcome the barrier.

Quantum dynamics. Using an efficient exact diagonalization method [45] we are able to simulate the quantum dynamics and observe mean-field signatures of the DPT that survive quantum noise. For example, in Fig. 4(a) we probe a typical time trace of $\left\langle\hat{S}_{z}\right\rangle$ in the resonant regime [same as Fig. 2(a)] for experimentally realistic $N=40,200,600$ and find that it is possible to gradually observe the signature retrapping dynamics with increasing $N$, before quantum fluctuations dephase $\left\langle\hat{S}_{z}\right\rangle \rightarrow 0$.

Conversely, pure quantum measures such as entanglement and quantum correlations also show clear signatures of the DPT, chaos and nonintegrability. Figure 4(b) shows that the critical point of the DPT is signaled in the pronounced buildup of spin-boson entanglement in the untrapped phase, quantified by the entanglement entropy $S_{\mathrm{vN}}=-\operatorname{Tr}\left[\hat{\rho}_{s} \ln \left(\hat{\rho}_{s}\right)\right]$, where $\hat{\rho}_{s}$ is the reduced density matrix of the spins. For $\delta / \Omega \lesssim$ 1 the time-averaged entropy $\overline{S_{\mathrm{vN}}}=(1 / T) \int_{t_{0}}^{T+t_{0}} d t S_{\mathrm{vN}}(t)$ demarcates the trapped and untrapped phases consistent with the order parameter $\overline{S_{z}}$, with $\overline{S_{\mathrm{vN}} \text { trapped }} \ll \overline{S_{\mathrm{VN}} \text { untrapped. The }}$ entanglement decreases with increasing $\delta / \Omega$ as the bosons become eliminated, although we expect that, e.g., bipartite entanglement between the spins will retain indications of the DPT. Panel (c) also demonstrates that the temporal fluctuations of the entanglement vary with the underlying integrability of the Hamiltonian $[53,54]$. Comparing to the Lyapunov exponent in Fig. 2, we see a correlation between regions of chaotic (nonintegrable) dynamics and suppressed temporal fluctuations of $S_{\mathrm{vN}}(t)$ (relative to $\overline{S_{\mathrm{vN}}}$ ).

Panel (d) evidences that the critical region of the DPT can be diagnosed by the rapid growth of quantum fluctuations. By empirically fitting $\left\langle\left(\Delta \hat{S}_{z}\right)^{2}\right\rangle=\left\langle\hat{S}_{z}^{2}\right\rangle-\left\langle\hat{S}_{z}\right\rangle^{2} \propto\left(1-e^{-\gamma t}\right)$ we find that the rate at which quantum fluctuations buildup, characterized by $\gamma$, is largest for $\tilde{g} \sim \tilde{g}_{\text {DPT }}$. This is consistent with the effective potential description, as near the critical coupling the mean-field trajectory spends an increasing amount of time probing the central barrier (e.g., unstable fixed point) $[8,45,55,56]$, motivating the expectation that quantum fluctuations will grow exponentially. Nevertheless, we also note that a similarly rapid growth of fluctuations is observed away from the DPT for $1 \lesssim \delta / \Omega \lesssim 2$ as a consequence of classical chaos [30], indicating that it is important to carefully differentiate the effects of chaos from the DPT in quantum dynamics.

Experimental realization. The full dynamical phase diagram of the Dicke model can be studied in a range of current state-of-the-art AMO quantum simulators, but most readily in arrays of trapped ions. In particular, the Dicke model was recently realized in a 2D Penning trap configuration [38,39] where a spin $1 / 2$ is encoded in two internal states of each ion and the collective center-of-mass motional mode of the ion crystal realizes the bosonic degree of freedom. A pair of lasers generates an optical dipole force that couples the internal state of each ion to the motional degree of freedom [57]. A transverse field is generated by applying microwaves with tunable strength $\Omega$ that coherently drive the two internal levels and the spin-boson coupling $g$ can be in principle controlled via the applied laser power. Moreover, $\delta$ can be varied by controlling the detuning of the lasers relative to the frequency of the targeted center-of-mass mode [57]. Considering the simulator reported in Refs. [38,39], we predict it should be possible to probe regimes $0.1 \lesssim \delta / \Omega \lesssim 10$ for $\tilde{g} \sim 1$ on timescales that are fast compared to relevant sources of single-particle decoherence of the spins $[45,46,58]$. In contrast to related studies of the Dicke model in optical cavities, the trapped ion simulator has little damping of the bosonic mode and thus to an excellent approximation can be neglected. Further, ion crystals of $N \approx 200$ [58] are possible in the 2D geometry, which is sufficient to access the signatures of the DPT as in Figs. 1 and 4 [45]. Looking ahead, implementing the Dicke model in 3D ion crystals [59] would open a path to even larger system sizes that are beyond the capability of numerical methods.

Conclusion. We have studied a DPT in the Dicke model and unique features arising from nonintegrable and chaotic regimes of parameter space. Our numerical study indicates signatures of the DPT survive in the quantum dynamics and are accessible in current state-of-the-art AMO quantum simulators based on, e.g., trapped-ion arrays. This can 
motivate future investigations connecting to quantum phenomena such as, e.g., information scrambling [30,60]. Additionally, studying entanglement dynamics associated with DPTs in collective systems will open new directions for the generation of metrologically useful states for quantumenhanced sensors [61] or frequency and time standards [62].

\section{ACKNOWLEDGMENTS}

We acknowledge helpful discussions with Jamir Marino and Matthew Affolter regarding the manuscript. Recently,
Armando Relaño and Miguel A. Bastarrachea Magnani pointed out the correspondence of the DPT with emergent EQPTs in the boson-dominated regime and we are thankful for their contribution. This work is supported by the AFOSR Grant No. FA9550-18-1-0319, by the DARPA and ARO Grant No. W911NF-16-1-0576, the ARO single investigator award W911NF-19-1-0210, and the NSF PHY1820885, NSF JILAPFC PHY-1734006, and NSF QLCI-2016244 grant by the DOE, Office of Science, National Quantum Information Science Research Centers, Quantum Systems Accelerator (QSA) and by NIST.
[1] M. Heyl, A. Polkovnikov, and S. Kehrein, Dynamical Quantum Phase Transitions in the Transverse-Field Ising Model, Phys. Rev. Lett. 110, 135704 (2013).

[2] M. Schiró and M. Fabrizio, Time-Dependent Mean Field Theory for Quench Dynamics in Correlated Electron Systems, Phys. Rev. Lett. 105, 076401 (2010).

[3] B. Sciolla and G. Biroli, Dynamical transitions and quantum quenches in mean-field models, J. Stat. Mech.: Theory Exp. (2011) P11003.

[4] J. C. Halimeh, V. Zauner-Stauber, I. P. McCulloch, I. de Vega, U. Schollwöck, and M. Kastner, Prethermalization and persistent order in the absence of a thermal phase transition, Phys. Rev. B 95, 024302 (2017).

[5] J. C. Halimeh and V. Zauner-Stauber, Dynamical phase diagram of quantum spin chains with long-range interactions, Phys. Rev. B 96, 134427 (2017).

[6] B. Žunkovič, M. Heyl, M. Knap, and A. Silva, Dynamical Quantum Phase Transitions in Spin Chains with Long-Range Interactions: Merging Different Concepts of Nonequilibrium Criticality, Phys. Rev. Lett. 120, 130601 (2018).

[7] A. Lerose, J. Marino, B. Žunkovič, A. Gambassi, and A. Silva, Chaotic Dynamical Ferromagnetic Phase Induced by Nonequilibrium Quantum Fluctuations, Phys. Rev. Lett. 120, 130603 (2018).

[8] A. Lerose, B. Žunkovič, J. Marino, A. Gambassi, and A. Silva, Impact of nonequilibrium fluctuations on prethermal dynamical phase transitions in long-range interacting spin chains, Phys. Rev. B 99, 045128 (2019).

[9] M. Schiró and M. Fabrizio, Quantum quenches in the Hubbard model: Time-dependent mean-field theory and the role of quantum fluctuations, Phys. Rev. B 83, 165105 (2011).

[10] F. Peronaci, M. Schiró, and M. Capone, Transient Dynamics of $d$-Wave Superconductors after a Sudden Excitation, Phys. Rev. Lett. 115, 257001 (2015).

[11] B. Sciolla and G. Biroli, Quantum quenches, dynamical transitions, and off-equilibrium quantum criticality, Phys. Rev. B 88, 201110(R) (2013).

[12] A. Chiocchetta, M. Tavora, A. Gambassi, and A. Mitra, Shorttime universal scaling in an isolated quantum system after a quench, Phys. Rev. B 91, 220302(R) (2015).

[13] A. Chiocchetta, A. Gambassi, S. Diehl, and J. Marino, Dynamical Crossovers in Prethermal Critical States, Phys. Rev. Lett. 118, 135701 (2017).

[14] M. Eckstein, M. Kollar, and P. Werner, Thermalization after an Interaction Quench in the Hubbard Model, Phys. Rev. Lett. 103, 056403 (2009).
[15] A. Gambassi and P. Calabrese, Quantum quenches as classical critical films, Europhys. Lett. 95, 66007 (2011).

[16] P. Smacchia, M. Knap, E. Demler, and A. Silva, Exploring dynamical phase transitions and prethermalization with quantum noise of excitations, Phys. Rev. B 91, 205136 (2015).

[17] J. Lang, B. Frank, and J. C. Halimeh, Concurrence of dynamical phase transitions at finite temperature in the fully connected transverse-field Ising model, Phys. Rev. B 97, 174401 (2018).

[18] S. Smale, P. He, B. A. Olsen, K. G. Jackson, H. Sharum, S. Trotzky, J. Marino, A. M. Rey, and J. H. Thywissen, Observation of a transition between dynamical phases in a quantum degenerate Fermi gas, Sci. Adv. 5, eaax1568 (2019).

[19] H.-X. Yang, T. Tian, Y.-B. Yang, L.-Y. Qiu, H.-Y. Liang, A.-J. Chu, C. B. Dağ, Y. Xu, Y. Liu, and L.-M. Duan, Observation of dynamical quantum phase transitions in a spinor condensate, Phys. Rev. A 100, 013622 (2019).

[20] T. Tian, H.-X. Yang, L.-Y. Qiu, H.-Y. Liang, Y.-B. Yang, Y. Xu, and L.-M. Duan, Observation of Dynamical Quantum Phase Transitions with Correspondence in an Excited State Phase Diagram, Phys. Rev. Lett. 124, 043001 (2020).

[21] J. A. Muniz, D. Barberena, R. J. Lewis-Swan, D. J. Young, J. R. K. Cline, A. M. Rey, and J. K. Thompson, Exploring dynamical phase transitions with cold atoms in an optical cavity, Nature (London) 580, 602 (2020).

[22] J. Zhang, G. Pagano, P. W. Hess, A. Kyprianidis, P. Becker, H. Kaplan, A. V. Gorshkov, Z.-X. Gong, and C. Monroe, Observation of a many-body dynamical phase transition with a 53-qubit quantum simulator, Nature (London) 551, 601 (2017).

[23] R. H. Dicke, Coherence in spontaneous radiation processes, Phys. Rev. 93, 99 (1954).

[24] B. M. Garraway, The Dicke model in quantum optics: Dicke model revisited, Philos. Trans. R. Soc. London A 369, 1137 (2011).

[25] P. Kirton, M. M. Roses, J. Keeling, and E. G. Dalla Torre, Introduction to the Dicke model: From equilibrium to nonequilibrium, and vice versa, Adv. Quantum Technol. 2, 1800043 (2019).

[26] A. Altland and F. Haake, Equilibration and macroscopic quantum fluctuations in the Dicke model, New J. Phys. 14, 073011 (2012).

[27] A. Altland and F. Haake, Quantum Chaos and Effective Thermalization, Phys. Rev. Lett. 108, 073601 (2012).

[28] W. Buijsman, V. Gritsev, and R. Sprik, Nonergodicity in the Anisotropic Dicke Model, Phys. Rev. Lett. 118, 080601 (2017).

[29] J. Chávez-Carlos, M. A. Bastarrachea-Magnani, S. LermaHernández, and J. G. Hirsch, Classical chaos in atom-field systems, Phys. Rev. E 94, 022209 (2016). 
[30] R. Lewis-Swan, A. Safavi-Naini, J. J. Bollinger, and A. M. Rey, Unifying scrambling, thermalization and entanglement through measurement of fidelity out-of-time-order correlators in the Dicke model, Nat. Commun. 10, 1 (2019).

[31] D. Villaseñor, S. Pilatowsky-Cameo, M. A. BastarracheaMagnani, S. Lerma-Hernández, L. F. Santos, and J. G. Hirsch, Quantum vs classical dynamics in a spin-boson system: Manifestations of spectral correlations and scarring, New J. Phys. 22, 063036 (2020).

[32] C. Emary and T. Brandes, Quantum Chaos Triggered by Precursors of a Quantum Phase Transition: The Dicke Model, Phys. Rev. Lett. 90, 044101 (2003).

[33] C. Emary and T. Brandes, Chaos and the quantum phase transition in the Dicke model, Phys. Rev. E 67, 066203 (2003).

[34] P. Pérez-Fernández, A. Relaño, J. M. Arias, P. Cejnar, J. Dukelsky, and J. E. García-Ramos, Excited-state phase transition and onset of chaos in quantum optical models, Phys. Rev. E 83, 046208 (2011).

[35] T. Brandes, Excited-state quantum phase transitions in Dicke superradiance models, Phys. Rev. E 88, 032133 (2013).

[36] R. Puebla and A. Relaño, Non-thermal excited-state quantum phase transitions, Europhys. Lett. 104, 50007 (2014).

[37] M. A. Bastarrachea-Magnani, S. Lerma-Hernández, and J. G. Hirsch, Comparative quantum and semiclassical analysis of atom-field systems. I. Density of states and excited-state quantum phase transitions, Phys. Rev. A 89, 032101 (2014).

[38] A. Safavi-Naini, R. J. Lewis-Swan, J. G. Bohnet, M. Gärttner, K. A. Gilmore, J. E. Jordan, J. Cohn, J. K. Freericks, A. M. Rey, and J. J. Bollinger, Verification of a Many-Ion Simulator of the Dicke Model Through Slow Quenches across a Phase Transition, Phys. Rev. Lett. 121, 040503 (2018).

[39] J. Cohn, A. Safavi-Naini, R. J. Lewis-Swan, J. G. Bohnet, M. Gärttner, K. A. Gilmore, J. E. Jordan, A. M. Rey, J. J. Bollinger, and J. K. Freericks, Bang-bang shortcut to adiabaticity in the Dicke model as realized in a Penning trap experiment, New J. Phys. 20, 055013 (2018).

[40] K. Baumann, C. Guerlin, F. Brennecke, and T. Esslinger, Dicke quantum phase transition with a superfluid gas in an optical cavity, Nature (London) 464, 1301 (2010).

[41] J. Klinder, H. Keßler, M. Wolke, L. Mathey, and A. Hemmerich, Dynamical phase transition in the open Dicke model, Proc. Natl. Acad. Sci. USA 112, 3290 (2015).

[42] Z. Zhang, C. H. Lee, R. Kumar, K. J. Arnold, S. J. Masson, A. L. Grimsmo, A. S. Parkins, and M. D. Barrett, Dicke-model simulation via cavity-assisted Raman transitions, Phys. Rev. A 97, 043858 (2018).

[43] R. M. Kroeze, Y. Guo, V. D. Vaidya, J. Keeling, and B. L. Lev, Spinor Self-Ordering of a Quantum Gas in a Cavity, Phys. Rev. Lett. 121, 163601 (2018).

[44] R. Puebla, Finite-component dynamical quantum phase transitions, Phys. Rev. B 102, 220302(R) (2020).

[45] See Supplemental Material at http://link.aps.org/supplemental/ 10.1103/PhysRevResearch.3.L022020 for analytic calculation of dynamical critical points in SR and BDR, analysis of connection between the DPT and EQPT, extended data from numerical simulations and a discussion of parameters for a proposed experimental realization.
[46] A. Safavi-Naini, R. J. Lewis-Swan, J. G. Bohnet, M. Garttner, K. A. Gilmore, E. Jordan, J. Cohn, J. K. Freericks, A. M. Rey, and J. J. Bollinger, Exploring adiabatic quantum dynamics of the Dicke model in a trapped ion quantum simulator, arXiv:1711.07392.

[47] S. Strogatz, Nonlinear Dynamics and Chaos: With Applications to Physics, Biology, Chemistry, and Engineering, Studies in Nonlinearity (CRC, Boca Raton, FL, 2000).

[48] I. Sainz, A. B. Klimov, and L. Roa, Quantum phase transitions in an effective Hamiltonian: Fast and slow systems, J. Phys. A: Math. Theor. 41, 355301 (2008).

[49] G. Liberti, F. Plastina, and F. Piperno, Scaling behavior of the adiabatic Dicke model, Phys. Rev. A 74, 022324 (2006).

[50] J. Keeling, M. J. Bhaseen, and B. D. Simons, Collective Dynamics of Bose-Einstein Condensates in Optical Cavities, Phys. Rev. Lett. 105, 043001 (2010).

[51] M. A. Bastarrachea-Magnani, A. Relaño, S. Lerma-Hernández, B. L. del Carpio, J. Chávez-Carlos, and J. G. Hirsch, Adiabatic invariants for the regular region of the Dicke model, J. Phys. A: Math. Theor. 50, 144002 (2017).

[52] A. Relaño, M. A. Bastarrachea-Magnani, and S. LermaHernández, Approximated integrability of the Dicke model, Europhys. Lett. 116, 50005 (2016).

[53] H. B. Kaplan, L. Guo, W. L. Tan, A. De, F. Marquardt, G. Pagano, and C. Monroe, Many-Body Dephasing in a TrappedIon Quantum Simulator, Phys. Rev. Lett. 125, 120605 (2020).

[54] T. Kiendl and F. Marquardt, Many-Particle Dephasing after a Quench, Phys. Rev. Lett. 118, 130601 (2017).

[55] A. Lerose and S. Pappalardi, Bridging entanglement dynamics and chaos in semiclassical systems, Phys. Rev. A 102, 032404 (2020).

[56] A. Lerose and S. Pappalardi, Origin of the slow growth of entanglement entropy in long-range interacting spin systems, Phys. Rev. Research 2, 012041(R) (2020).

[57] J. W. Britton, B. C. Sawyer, A. C. Keith, C.-C. J. Wang, J. K. Freericks, H. Uys, M. J. Biercuk, and J. J. Bollinger, Engineered two-dimensional Ising interactions in a trapped-ion quantum simulator with hundreds of spins, Nature (London) $\mathbf{4 8 4}, 489$ (2012).

[58] J. G. Bohnet, B. C. Sawyer, J. W. Britton, M. L. Wall, A. M. Rey, M. Foss-Feig, and J. J. Bollinger, Quantum spin dynamics and entanglement generation with hundreds of trapped ions, Science 352, 1297 (2016).

[59] A. Mortensen, E. Nielsen, T. Matthey, and M. Drewsen, Observation of Three-Dimensional Long-Range Order in Small Ion Coulomb Crystals in an rf Trap, Phys. Rev. Lett. 96, 103001 (2006).

[60] M. K. Joshi, A. Elben, B. Vermersch, T. Brydges, C. Maier, P. Zoller, R. Blatt, and C. F. Roos, Quantum Information Scrambling in a Trapped-Ion Quantum Simulator with Tunable Range Interactions, Phys. Rev. Lett. 124, 240505 (2020).

[61] K. A. Gilmore, J. G. Bohnet, B. C. Sawyer, J. W. Britton, and J. J. Bollinger, Amplitude Sensing Below the Zero-Point Fluctuations with a Two-Dimensional Trapped-Ion Mechanical Oscillator, Phys. Rev. Lett. 118, 263602 (2017).

[62] S. M. Brewer, J.-S. Chen, A. M. Hankin, E. R. Clements, C. W. Chou, D. J. Wineland, D. B. Hume, and D. R. Leibrandt, ${ }^{27} \mathrm{Al}^{+}$ Quantum-Logic Clock with a Systematic Uncertainty Below $10^{-18}$, Phys. Rev. Lett. 123, 033201 (2019). 